\title{
Correction to: Lung ultrasound to predict pediatric intensive care admission in infants with bronchiolitis (LUSBRO study)
}

\author{
Sara Bobillo-Perez ${ }^{1,2} \cdot$ Clara Sorribes $^{3} \cdot$ Paula Gebellí $^{3} \cdot$ Nuria Lledó $^{3} \cdot$ Marta Castilla $^{3} \cdot$ Miquel Ramon $^{3}$. \\ Javier Rodriguez-Fanjul ${ }^{4}$
}

Published online: 27 February 2021

(C) Springer-Verlag GmbH Germany, part of Springer Nature 2021

\section{Correction to: European Journal of Pediatrics https://doi.org/10.1007/s00431-021-03978-4}

The author names on the authorship section of the published version of the above article were interchanged. The names are now presented correctly above. The original article has been corrected.

Publisher's note Springer Nature remains neutral with regard to jurisdictional claims in published maps and institutional affiliations.

The online version of the original article can be found at https://doi.org/ 10.1007/s00431-021-03978-4

Javier Rodriguez-Fanjul

javier.rodriguez.fanjul@gmail.com

Sara Bobillo-Perez

sbobillo@sjdhospitalbarcelona.org

Clara Sorribes

sorribes.clara@gmail.com

Paula Gebellí

pgebelliv.hjd23.ics@gencat.cat

Nuria Lledó

nlledov.hjd23.ics@gencat.cat

Marta Castilla

mcastillav.hjd23.ics@gencat.cat
Miquel Ramon

mramonjimenez.hj23.ics@gencat.cat

1 Disorders of Immunity and Respiration of the Paediatric Critical Patient Research Group, Institut Recerca Hospital Sant Joan de Déu, Universitat de Barcelona, Barcelona, Spain

2 Paediatric Intensive Care Unit, Hospital Sant Joan de Déu, Universitat de Barcelona, Barcelona, Spain

3 Pediatric Intensive Care Unit, Paediatric Department, Hospital Joan XXIII, Tarragona, Spain

4 Neonatology Unit, Paediatric Department, Hospital Germans Trias I Pujol, Universitat Autònoma de Barcelona, Carretera de Canyet, s/n, 08916 Badalona, Barcelona, Spain 